\title{
Комплексная обработка оптического сигнала для магистральных волоконно-оптических линий связи
}

\author{
О.С. Сидельников ${ }^{*}$, А.С. Скидин, М.П. Федорук \\ Новосибирский государственный университет, г. Новосибирск \\ Институт вычислительных технологий СО РАН, г. Новосибирск \\ *E-mail: o.s.sidelnikov@gmail.com
}

DOI:10.31868/RFL2018.220-221

В настоящее время в волоконно-оптических линиях связи проблема преодоления ограничений, обусловленных нелинейными воздействиями, попрежнему является одним из ключевых вопросов, которые необходимо разрешить для дальнейшего повышения эффективности использования полосы пропускания канала. Для достижения данной цели в настоящий момент активно применяются технологии обработки оптического сигнала; в частности, активно исследуются нейронные сети в качестве вспомогательного метода, с помощью которого можно улучшить детектирование сигнала [1]. В данной работе исследуется потенциал использования нейронных сетей совместно с другими методами обработки сигнала.

Исследовалась схема передачи сигнала, показанная на рисунке 1. Передатчик создаёт 16-QАМ-сигнал и обрабатывает его (при необходимости) специальным методом модуляции, позволяющим регулировать в сигнале вероятности вхождения в него символов разной мощности [2]. Линия состоит из 10 пролётов, каждый пролёт включает в себя 100 км волокна, а также усилитель с уровнем шума 4.5 дБ. На приёмнике компенсируется хроматическая дисперсия, фазовый сдвиг, а также с помощью статической нейронной сети компенсируются нелинейные воздействия. Структура нейронной сети показана на рисунке 2. Входные символы разделяются на действительную и мнимую части, сеть состоит из входного слоя, двух скрытых слоёв по 16 нейронов и выходного слоя.

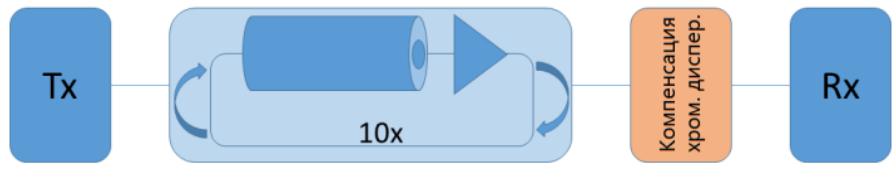

Рис. 1. Схема исследуемой линии

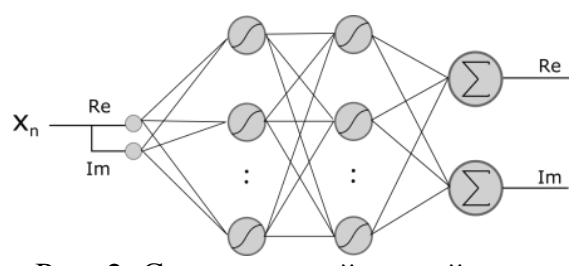

Рис. 2. Структура нейронной сети.

При математическом моделировании распространения сигнала по оптическому волокну использовалось нелинейное уравнение Шрёдингера (NLSE) [3], которое описывает эволюцию медленно меняющейся огибающей оптического сигнала $\mathrm{A}(\mathrm{z}, \mathrm{t})$ :

$$
\frac{\partial A}{\partial z}=-\frac{\alpha}{2} A-i \frac{\beta_{2}}{2} \frac{\partial^{2} A}{\partial t^{2}}+i \gamma|A|^{2} A .
$$


Данное уравнение решалось численно с помощью симметричного метода Фурье расщепления по физическим процессам со следующими параметрами: $\alpha=0.2$ дБ/км, нелинейность волокна $\gamma=1.41 /($ Вт·км), хроматическая дисперсия $\beta_{2}=-25$ пс $2 /$ м, длина волны $\lambda=1550$ нм, количество отсчётов на период $q=16$.

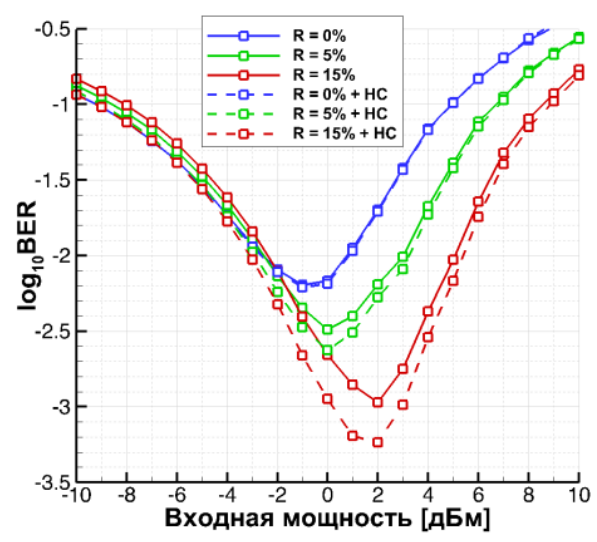

Рис. 3. Зависимость BER от различных конфигураций обработки сигнала.

На рисунке 3 показана полученная зависимость между частотой битовых ошибок и различными конфигурациями схем обработки сигнала. Мера вводимых ограничений оценивается через величину $R$, которая определяется как величина уменьшения номинальной скорости передачи данных относительно скорости передачи формата 16-QАМ без использования специальной модуляции. Ключевой особенностью данных результатов является то, что в отсутствие специальных методов модуляции статическая нейронная сеть работает с таким же качеством, с которым работают традиционные детекторы. При этом, когда используется регулирование вероятностей появления символов, которые на сигнальном созвездии соответствуют высокой мощности, статическая нейронная сеть начинает давать дополнительное улучшение, не связанное само по себе с улучшением, вызванным специальной модуляцией [2].

Данное обстоятельство можно объяснить как с позиции нейронных сетей, так и с позиций физических эффектов. С позиции нейронных сетей улучшение работы статической сети в канале с памятью возможно тогда, когда память канала «уменьшается», что и происходит при применении специальной модуляции, поскольку она направлена на компенсацию нелинейных эффектов, обуславливающих наличие памяти у канала. Для описания улучшения с физической точки зрения стоит отметить, что из уравнения Шрёдингера можно получить отдельные два уравнения эволюции фазы $\rho(\mathrm{z}, \mathrm{t})$ и амплитуды сигнала $\mathrm{a}(\mathrm{z}, \mathrm{t})[4]$ :

$$
\begin{aligned}
& \frac{\partial a}{\partial z}=-\frac{\alpha}{2} a+\frac{\beta_{2}}{2}\left(2 \frac{\partial a}{\partial t} \frac{\partial \rho}{\partial t}+a \frac{\partial^{2} \rho}{\partial t^{2}}\right) \\
& a \frac{\partial \rho}{\partial z}=-\frac{\beta_{2}}{2}\left(\frac{\partial^{2} a}{\partial t^{2}}+a\left(\frac{\partial \rho}{\partial t}\right)^{2}\right)+\gamma a^{3} .
\end{aligned}
$$

Применение специальной модуляции в сигнале уменьшает дисперсию мгновенных значений производных амплитуды, что с точки зрения уравнения одновременно делает более «регулярным» эволюцию фазы, а также уменьшает влияние эволюции фазы на эволюцию амплитуды.

Работа Скидина А.С. (теоретический анализ результатов) выполнена при поддержке Российского научного фонда (проект №17-71-10118). Работа М.П. Федорука и О.С. Сидельникова выполнена при поддержке гранта 14.Y26.31.0017 Министерства образования и науки РФ.

\section{Литература}

[1] O.S. Sidelnikov et al. Quantum Electron. 47, 1147-1149 (2017).

[2] A.S. Skidin et al. Opt.Express 24, 30296-30308 (2016).

[3] G.P. Agrawal, Nonlinear fiber optics, 4th ed. Academic press, 2007, 539 c.

[4] M. Du et al. IEEE J. Quantum Electron., 31, 177-182 (1995). 